\title{
Minimum Clearance Time on the Prioritized Integrated Evacuation Network
}

\author{
Iswar Mani Adhikari ${ }^{1}$, Tanka Nath Dhamala ${ }^{2,}$ * \\ ${ }^{1}$ Prithvi Narayan Campus, Tribhuvan University, Pokhara, Nepal \\ ${ }^{2}$ Central Department of Mathematics, Tribhuvan University, Kathmandu, Nepal \\ Email address: \\ adhikariim35@gmil.com (I. M. Adhikari),amb.dhamala@daadindia.org (T. N. Dhamala) \\ ${ }^{*}$ Corresponding author
}

To cite this article:

Iswar Mani Adhikari, Tanka Nath Dhamala. Minimum Clearance Time on the Prioritized Integrated Evacuation Network. American Journal of Applied Mathematics. Vol. 8, No. 4, 2020, pp. 207-215. doi: 10.11648/j.ajam.20200804.15

Received: April 8, 2020; Accepted: July 20, 2020; Published: July 28, 2020

\begin{abstract}
The evacuation planning problem can be viewed as different variants of dynamic flow maximization and time minimization problems. An optimal solution to the latter problem sends a given amount of flow from disaster zones to safe zones in minimum time. We solve this problem on an embedded integrated network of a prioritized primary and a bus-routed secondary sub-networks. For a lexicographically maximum (lex-max) dynamic flow problem, we are given a time horizon and a prioritized network, where we need a feasible dynamic flow that lexicographically maximizes the flow amount leaving each terminal respecting the priority. Here, we use the quickest transshipment partial arc reversal strategy to collect the evacuees in minimum time from the disaster zones to the pickup locations of the primary sub-network. By treating such pickup locations as sources, the available set of transit-buses is assigned in the secondary sub-network to shift the evacuees finally to the sinks on the first-come-first-serve basis. This novel approach proposed here is better suited for the simultaneous flow of evacuees with minimum waiting delay at such pickup locations in the integrated evacuation network topology. The lane reversal strategy significantly reduces the evacuation time, whereas reversing them only partially has an additional benefit that the unused road capacities can be used for supplying emergency logistics and allocating facilities as well.
\end{abstract}

Keywords: Evacuation Planning, Integrated Network, Minimum Clearance Time, Lexicographic Flow, Partial Arc Reversal

\section{Introduction}

Problems on dynamic networks were firstly introduced by Ford and Fulkerson $[1,2]$. The minimum clearance time evacuation planning problem considered here has been modeled in dynamic networks in which each arc has a nonnegative flow capacity and integer transit time. This is one of the fundamental problems in evacuation planning to find the minimum time limit such that all supplies can be sent to the sinks (safe places) from the sources (disastrous zones). There has been a fair amount of work regarding different aspects of the evacuation planning problems, including the quickest one for minimum clearance time, as referred by [36]. These problems are usually handled with a different perspective, namely, the transit-based, car-based, and pedestrian movements depending on evacuation scenarios. The transit-based planning problems are to minimize the duration of evacuation by routing and scheduling a fleet of vehicles, say buses. The NP-hard multi-depot, multi-trip busbased evacuation planning problem (BEPP) was introduced and analyzed prominently in [7] which is closer to the split delivery multi-depot vehicle routing problem with inter-depot routes. However, if there is only one bus-depot, assuming that the bus pick ups the same number of people that equals its capacity, the author in [8] has also proposed the BEPP for the evacuation of a region from a set of collection points to a set of capacitated sinks. Based on such BEPP, Pyakurel et al. [9] explored a wide horizon to the research related to the transit-dependent evacuation planning problem. In their study, the excess exterior points of the disaster region were taken as the pickup locations and the available open spaces as the sinks. Evacuees were supposed to gather themselves from their residents to the nearby pickup locations. Homogeneous buses with the uniform capacity were used for the evacuees' pickup. Their computational analysis noticed that the domain of optimal solutions remains on the larger number of buses with higher capacity and speed irrespective of the number of 
sources and sinks chosen.

A solution to maximum flow problem sends the maximal amount of flow from the sources to the sinks for the fixed integer time horizon T. Lexicographically maximum (lexmax) flow problem with many sources and many sinks was introduced and many efficient algorithms were presented from different aspects in [10-13]. In such a problem, the terminals (sources and/or sinks) are ordered with certain priority for a lex-max flow respecting the priority. Such a lex-max flow is not necessarily a maximum flow and vice versa, however, they are equivalent for two-terminal networks [2]. If a flow is both source-optimal and sinkoptimal, then it becomes the lexicographically optimal flow in the priority network [14].

The lex-max dynamic contraflow problem was investigated in [15] where reversals of arcs are allowed. The partial contraflow with path reversals leads to a significant improvement in increasing the flow values, decreasing the evacuation time, and utilization of the unused capacities of paths for humanitarian logistics and vehicle movements, [16]. Authors in [17] have introduced the partial lane reversal strategy and presented efficient dynamic flow algorithms for quickest and maximum flow evacuation planning problems. In the quickest transshipment problem, a given number of evacuees has to be shifted in minimum time. Such problems have been studied by the help of the lex-max dynamic flow problem applying the minimum cost flow computations as a tool [18]. An algorithm to find the universally quickest transshipment has been presented [19]. They also have used the minimum cost flow computations.

In this work, evacuees are collected from the disaster zone to the pickup locations of the primary sub-network in minimum time as the quickest transshipment by using the lex-max flow approach. Considering such pickup locations as the sources, the available set of transit-buses are also assigned in the network to evacuate the evacuees safely to the sinks on the first-come-first-serve basis. This novel approach proposed here is better suited for the simultaneous flow of evacuees with minimum waiting delay at such pickup locations for the evacuation planning problem in the integrated network.

In Section 2, we explain an integrated network topology. The arrival of evacuees at the primary sub-network in Section 3. The assignment of vehicles in the secondary subnetwork including its embedding to the primary sub-network is described in Section 4. An integrated solution approach for the proposed problem is presented in Section 5. Section 6 concludes the paper.

\section{An Integrated Network Topology}

Consider two separate dynamic networks $\mathrm{N}_{1}$ and $\mathrm{N}_{2}$ as a primary and a secondary sub-network, respectively. These two are embedded as a unit to form an integrated evacuation network as $N=\mathrm{N}_{1} \cup \mathrm{N}_{2}$. Among them, $N_{1}$ is a directed twoway network and $N_{2}$ is the mixed network having directed one-way arcs and undirected edges.

\subsection{The Primary Sub-network}

Let $N_{1}=\left(S, V, A, u_{a}, \tau_{a}, Y\right)$ be a primary sub-network, $S=\left\{s_{1}, s_{2}, \ldots, s_{n}\right\} \quad, \quad V=\left(v_{1}, v_{2}, \ldots, v_{n}\right) \quad$ and $Y=\left(y_{1}, y_{2}, \ldots, y_{n}\right)$ denote the sets of sources, auxiliary nodes, and pickup locations, respectively. The set of arcs joining any two nodes in $N_{1}$ are denoted by $A$ where the capacity and transit time are denoted by $u_{a}$ and $\tau_{a}$, respectively. The capacity $u_{a}: A \rightarrow \mathbb{Z}_{\geq 0}$ restricts the amount of flow on the arc and the transit time $\tau_{a}: A \rightarrow \mathbb{Z}_{\geq 0}$ represents the time required for the flow to transverse through the respective arc. During evacuee arrival at the pickup location $Y$ from $S$, the set $Y=\cup\left\{Y_{i}\right\}$ is the set of sinks.

\subsection{The Secondary Sub-network}

Let $N_{2}=\left(d, Y, E, \tau_{e}, Z\right)$ be a secondary sub-network, where $d$ and $Z=\left\{z_{1}, z_{2}, \ldots, z_{n}\right\}$ are the bus depot and sink, respectively. A set of transit-buses $B=\left\{b_{1}, b_{2}, \ldots, b_{n}\right\}$ with uniform capacity are located initially at the bus depot $d$ and are assigned as required during the evacuation procedure. Buses do not return to $d$ even after the completion of the evacuation process as it is risky to return to it under such threats. So, it does not play significant roles further in the system. The set $E$ consists of the one-way $\operatorname{arcs} e=(d, y)$ with $y \in Y$ and the undirected edges $e=[y, z]$, with $y \in Y$ and $z \in Z$. Transit times of the respective arcs and edges are denoted by $\tau_{e} \in \mathbb{Z}_{+}$as $\tau_{d i}$ and $\tau_{i j}$, respectively.

\subsection{An Embedding of the Integrated Network}

In an embedding, $N=N_{1} \cup N_{2}$, capacity, transit times, and other related attributes for $N$ are carried over from their respective sub-networks. The node $Y$ works as the sinks concerning $N_{1}$ but as the supply for $N_{2}$. Transit-buses are assigned in $N_{2}$ from $d$, which are sufficiently closer to it, on the first-come-first-serve basis, i.e., the evacuees collected earlier will be assigned earlier to the appropriate sink and will be continued till the supply is available at $\mathrm{Y}$ respecting the capacity of the sinks $Z$.

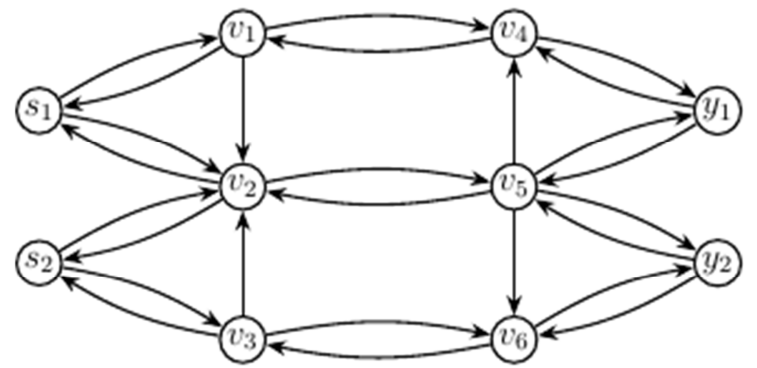

(i)

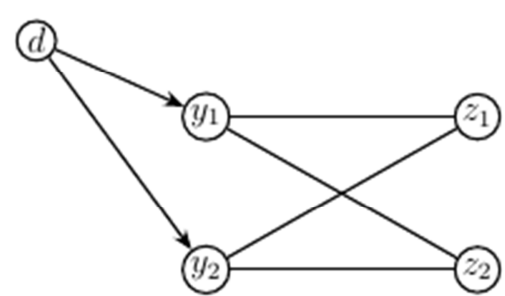

(ii)

Figure 1. (i) A primary sub-network, and (ii) a secondary sub-network. 


\section{Arrival of Evacuees}

The nature of the evacuees and the intensity of the disasters may vary at different sources. Mostly, the evacuees collected at pickup locations have to wait long for being assigned to transit-buses in the network. On the other hand, the nature of the pickup locations may also differ from each other depending upon their locations, availability, etc. So, the network is better to be prioritized. For such formulations, the lexicographic approach is better suited as different priorities can be assigned to various attributes. Such an approach can be applied to achieve the quickest transshipment with several sources and sinks, provided with the given supply and demand [18].

An $s-y$ flow of evacuees over time is a non-negative function $f$ on $A \times T$ for given time $T=\{0,1, \ldots, T\}$ satisfying the flow conservation and capacity constraints (1-3). The inequality flow conservation constraints allow it to wait for flow at intermediate nodes, however, the equality flow conservation constraints force that flows entering an intermediate node must leave it.

$$
\begin{aligned}
& \sum_{a \epsilon A_{i}^{i n}} \sum_{\sigma=\tau_{a}}^{T} f\left(a, \sigma-\tau_{a}\right)-\sum_{a \epsilon A_{i}^{o u t}} \sum_{\sigma=0}^{T} f(a, \sigma)= \\
& 0, \forall i \in V \backslash(S \cup Y) \\
& \sum_{a \epsilon A_{i}^{i n}} \sum_{\sigma=\tau_{a}}^{\theta} f\left(a, \sigma-\tau_{a}\right)-\sum_{a \epsilon A_{i}^{\text {out }}} \sum_{\sigma=0}^{\theta} f(a, \sigma) \geq \\
& 0, \forall i \in V \backslash(S \cup Y), \theta \in T \\
& 0 \leq \mathrm{f}(\mathrm{a}, \theta) \leq u_{a} \forall a \in A, \theta \in T
\end{aligned}
$$

The sets of outgoing and incoming arcs for the node $i \in V$ are denoted by, $A_{i}^{\text {out }}=\{a=(i, j) \in A\}$ and $A_{i}^{\text {in }}=\{a=$ $(j, i) \in A\}$, respectively. Not stated otherwise, for all $y \in Y$ and $s \in S$, we assume that $A_{i}^{\text {out }}=A_{i}^{\text {in }}=\phi$ in the case without arc reversals. However, for the source node $S$ and sink node $y$, we get the flow value be $v_{\mathrm{f}}(\mathrm{s})>0$ and $\mathrm{v}_{\mathrm{f}}(\mathrm{y})<0$, respectively, where $\sum_{i \epsilon V} \mathrm{v}_{\mathrm{f}}(\mathrm{i})=0$.

Problem 1. Given an evacuation sub-network $N_{1}=$ $\left(S, V, A, u_{a}, \tau_{a}, Y\right)$ with supplies at $S$, demands at $Y$ auxiliary nodes $V$, arc capacity $u_{a}$, and arc transit time $\tau_{a}$ for $a \in A$. The quickest partial arc reversal transshipment problem is to find the quickest arrival of evacuees at $Y$ with partial arc reversals capability.

Let the reversals of an arc $a=(i, j)$ be $a^{\prime}=(j, i)$. Then the transformed network of $N_{1}$ consists of the modified arc capacities and constant transit times as,

$u_{\bar{a}}=u_{a}+u_{a^{\prime}}$ and $\tau_{\bar{a}=} \tau_{a}$ if $a \in A$ and is $\tau_{a}$ for $a \notin A$. (4)

Here, an edge $\bar{a} \in \bar{A}$ in transformed network $\overline{N_{1}}$ if $a \vee a^{\prime} \in N_{1}$. Concerning the auxiliary reconfiguration, it is allowed to redirect the arc in any direction with the modified increased capacity but with the same transit time in either direction. The remaining graph structure and data are unaltered. In the transformed network $\overline{N_{1}}$, we have solved the lex-max dynamic flow problem on each arc as in [18] and saved all unused arc capacity as in [17].

Consider the set of sources and sinks be prioritized as $\left\{s_{1}, s_{2}, \ldots, s_{n}\right\}$ and $\left\{\mathrm{y}_{1}, \mathrm{y}_{2}, \ldots, \mathrm{y}_{\mathrm{k}}\right\}$, respectively. Let $s^{*}$ be the super source connected to such $s_{i}$ with arcs having the infinite capacity and zero transit time. Let the sinks in $N_{1}$ be prioritized with the highest priority to the nearest by determining their shortest distances concerning $s^{*}$. The lexmax flow within the specified time horizon $\mathrm{T}$ entering the sinks $Y$ in $N_{1}$ in that order can be computed in polynomialtime, as in Algorithm 1, based on [18].

Algorithm 1. Lex-max dynamic flow of evacuees in $N_{1}$.

Input: A dynamic sub-network $N_{1}=\left(S, V, A, u_{a}, \tau_{a}, Y\right)$. Let $V=V \cup\left\{s^{*}\right\}$ and $A^{\delta+1}=V \cup\left\{\left(s^{*}, s_{i}\right)\right\}: s_{i} \in S$, where $u_{a}\left(s^{*}, s_{i}\right)=\infty$ and $\tau_{a}\left(s^{*}, s_{i}\right)=0$ for $\left\{s^{*}\right\}$ be the super source. Let $N_{1}^{\delta+1}$ denotes the resulting network with $g_{0}^{\delta+1}=0$ be the zero flow and the set of chains be $\Gamma^{\delta+1}=$ $\phi$.

1. For $i=\delta, \ldots, 1$, set the arc be $A^{i}=A^{i+1}$,

a. If $s_{i}$ is sink, add the $\operatorname{arc} s_{i} s^{*}$ with $u_{a}\left(s^{*}, s_{i}\right)=\infty$ and $\tau_{a}\left(s^{*}, s_{i}\right)=-(T+1)$. Then get $f^{i}=$ minimum cost circulation in the resulting network using $\tau_{a}$ as the arc cost.

b. If $s_{i}$ is source, delete the $\operatorname{arc} s_{i} s^{*}$ from $A^{i}$ and get $f^{i}=$ minimum cost maximum $s^{*} s_{i}$ - flow in the resulting network using $\tau_{a}$ as the arc cost.

2. Update the dynamic flow $g^{i}=g^{i+1}+f^{i}$.

3. Let $\Delta^{i}=$ standard chain decomposition of $f^{i}$, then the chain decomposable set becomes $\Gamma^{i}=\Gamma^{i+1}+\Gamma^{i}$.

4. Finally, return $\Gamma=\Gamma^{1}$.

As a mutatis mutandis to the results in [18], we are here with two more results.

Theorem 1. Algorithm 1 constructed for the lex-max dynamic flow in $N_{1}$ gives the feasible solution for the lex-max number of evacuees at $Y$.

Theorem 2. For any lex-max dynamic flow problem, a lexmax dynamic flow can be computed via $k$ minimum cost flow $(M C F)$ computations in $O(k(M C F)(m, n))$, where $O(k(M C F)(m, n))=O(m \log n(m+n))[20]$.

Hoppe and Tardos in [18] studied the lex-max flow problem in dynamic networks by applying the MCF computations and have shown the quickest transshipment problem is equivalent to it. Different algorithms to solve such quickest transshipment have been presented based on the chain-decomposable flows and the minimization of submodular functions but are with high-order time complexity, though are polynomial-time solvable. Such chain decomposable flows help to generalize the stationary dynamic flows and the temporary repeated flows. The temporally repeated flows were referred to as the standard chain-decomposable flows. Such chain flows in a nonstandard chain decomposition may use a backwards arc with negative transit time. Based on this landmark paper [18], authors in [21] have also presented the quickest transshipment algorithm to determine the quickest transshipment as a convex combination of simple lex-max dynamic flows. Now, we are presenting the quickest partial arc reversal transshipment algorithm to get the quickest arrival of evacuees at $Y$ in $N_{1}$ corresponding to the arc reversal capability.

Algorithm 2. Quickest partial arc reversal transshipment algorithm. 
Input: A dynamic sub-network $N_{1}=\left(S, V, A, u_{a}, \tau_{a}, Y\right)$, with the supply and demand.

1. Construct a transformed dynamic sub-network $\overline{N_{1}}$ as in Equation (4).

2. Solve the quickest transshipment problem [18] in the transformed network of Step 1.

3. For each $\theta \in \mathrm{T}$ and reverse $a^{\prime} \in A$ up to capacity $c_{a}-u_{a}$ if and only if $c_{a}>u_{a}, u_{a}$ replaced by 0 whenever $a \notin A$, in $N_{1}$, where $c_{a}$ denotes the static $s-$ y flow value in each $a \in A$ for such sub-network.

4. For each $\theta \in \mathrm{T}$ and $a \in A$, if $a$ is reversed, $k_{a}=$ $u_{\bar{a}}-c_{a^{\prime}}$ and $k_{a \prime}=0$. If neither $a$ nor $a^{\prime}$ is reversed, $k_{a}=u_{a}-c_{a}$ where $k_{a}$ is saved capacity of $a$, [17].

5. Output: The quickest arrival of evacuees at $Y$ in $N_{1}$ with partial arc reversal capability.

Theorem 3. Algorithm 2 constructed for the quickest partial arc reversal transshipment gives the optimal solution for the quickest arrival of evacuees at $Y$ and saves the unused capacity.

Proof. The construction of a transformed dynamic network $\overline{N_{1}}$ for the given network $N_{1}$ in Step 1 is feasible. From the quickest transshipment problem [18], Step 2 is feasible. Moreover, the flow is either on arc $a$ or $a^{\prime}$ but not in both directions simultaneously. And such flow is not greater than the modified capacities of each arc in the transformed network. So, Step 3 is also feasible. Step 4 is also feasible and helps to compute the saved capacity by arc reversal capability. Hence, Algorithm 2 is feasible.

Now, we show that Algorithm 2 gives the optimal solution. In the transformed dynamic network $\overline{N_{1}}$ we compute the lex- max number of evacuees reached to each of the prioritized pickup locations $Y$ as the lex-max dynamic flow as in [18]. Moreover, the obtained solution is equivalent to the solution of the quickest arrival of evacuees at $Y$ in $N_{1}$ with the partial arc reversal up to the necessary capacity as in Step 3, [17]. Capacities of the arcs not used by the flow after partial arc reversals are computed in Step 4. $\square$

Theorem 4. For the quickest partial arc reversal transshipment in $N_{1}$, the quickest evacuee arrival problem can be computed in polynomial-time complexity via $k$ minimum cost flow (MCF) computations in $O(k(M C F)(m, n)) \quad$ time, where $\operatorname{MCF}(m, n)=O(m \log n(m+n \log n))$.

Proof. Steps 1, 3, and 4 related to the arc reversal capability as in Algorithm 2 are solved in linear time. So their time complexity is dominated by the time complexity of the computation of the quickest evacuee arrival in $N_{1}$ and is solved in polynomial-time in $\mathrm{O}(\mathrm{k}(\mathrm{MCF})(\mathrm{m}, \mathrm{n})$ where $M C F(m, n)=O(m \log n(m+n \log n))$ as in Theorem 2. $\square$

Example 1. Consider the primary sub-network for evacuee arrival as in Figure 2(i). Consider the evacuees at $s_{1}$ and $s_{2}$ be 16 and 13, respectively. Let the potential demands of $Y$ be $\alpha\left(Y_{1}\right)=20$ and $\alpha\left(Y_{2}\right)=9$. Consider $s^{*}$ be the super source connected to $s_{1}$ and $s_{2}$ in $N_{1}$ having infinite-capacity and zero-transit time, then the highest priority is to be assigned for $y_{1}$ as specified. Then the respective arrivals of the evacuees at $y_{1}$ and $y_{2}$ can be determined as in Table 1 (using Algorithm 1) and Table 2 (using Algorithm 2) with their respective paths assignment without and with arc reversal capability in $N_{1}$, respectively.

Table 1. The arrival of evacuees at $Y$ without partial arc reversal capability.

\begin{tabular}{|c|c|c|c|c|c|}
\hline Released time & Reached time & Paths assignment & Flow at $y_{1}$ & Flow at $y_{2}$ & Total flow \\
\hline 0 & 7 & $s_{1} \rightarrow v_{2} \rightarrow v_{5} \rightarrow v_{4} \rightarrow y_{1}$ & 2 & & 2 \\
\hline 1 & 8 & $s_{1} \rightarrow v_{2} \rightarrow v_{5} \rightarrow v_{4} \rightarrow y_{1}$ & 2 & & 4 \\
\hline 2 & 9 & $s_{1} \rightarrow v_{2} \rightarrow v_{5} \rightarrow v_{4} \rightarrow y_{1}$ & 2 & & 6 \\
\hline 3 & 10 & $s_{1} \rightarrow v_{2} \rightarrow v_{5} \rightarrow v_{4} \rightarrow y_{1}$ & 2 & & 8 \\
\hline 0 & 10 & $s_{2} \rightarrow v_{2} \rightarrow v_{5} \rightarrow y_{1}$ & 1 & & 9 \\
\hline 4 & 11 & $s_{1} \rightarrow v_{2} \rightarrow v_{5} \rightarrow v_{4} \rightarrow y_{1}$ & 2 & & 11 \\
\hline 1 & 11 & $s_{2} \rightarrow v_{2} \rightarrow v_{5} \rightarrow y_{1}$ & 1 & & 12 \\
\hline 5 & 12 & $s_{1} \rightarrow v_{2} \rightarrow v_{5} \rightarrow v_{4} \rightarrow y_{1}$ & 2 & & 14 \\
\hline 0 & 12 & $s_{2} \rightarrow v_{3} \rightarrow v_{6} \rightarrow y_{2}$ & - & 3 & 18 \\
\hline 6 & 13 & $s_{1} \rightarrow v_{2} \rightarrow v_{5} \rightarrow v_{4} \rightarrow y_{1}$ & 2 & & 20 \\
\hline 3 & 13 & $s_{2} \rightarrow v_{2} \rightarrow v_{5} \rightarrow y_{1}$ & 1 & & 21 \\
\hline 0 & 13 & $s_{1} \rightarrow v_{1} \rightarrow v_{4} \rightarrow v_{5} \rightarrow y_{1}$ & 2 & & 23 \\
\hline 1 & 13 & $s_{2} \rightarrow v_{3} \rightarrow v_{6} \rightarrow y_{2}$ & - & 3 & 26 \\
\hline 2 & 14 & $s_{2} \rightarrow v_{3} \rightarrow v_{6} \rightarrow y_{2}$ & - & 3 & 29 \\
\hline Total & & & 20 & 9 & 29 \\
\hline
\end{tabular}

Table 2. The arrival of evacuees at Y with partial arc reversal capability.

\begin{tabular}{lllll}
\hline Released time & Reached time & Paths assignment & Flow at $\boldsymbol{y}_{\mathbf{1}}$ & Flow at $\boldsymbol{y}_{\mathbf{2}}$ \\
\hline 0 & 7 & $s_{1} \rightarrow v_{2} \rightarrow v_{5} \rightarrow v_{4} \rightarrow y_{1}$ & 4 & 4 \\
1 & 8 & $s_{1} \rightarrow v_{2} \rightarrow v_{5} \rightarrow v_{4} \rightarrow y_{1}$ & 4 & \\
2 & 9 & $s_{1} \rightarrow v_{2} \rightarrow v_{5} \rightarrow v_{4} \rightarrow y_{1}$ & 4 & 12 \\
3 & 10 & $s_{1} \rightarrow v_{2} \rightarrow v_{5} \rightarrow v_{4} \rightarrow y_{1}$ & 4 & 16 \\
0 & 10 & $s_{2} \rightarrow v_{2} \rightarrow v_{5} \rightarrow y_{1}$ & 4 & 20 \\
0 & 12 & $s_{2} \rightarrow v_{3} \rightarrow v_{6} \rightarrow y_{2}$ & - & 26 \\
1 & 13 & $s_{2} \rightarrow v_{3} \rightarrow v_{6} \rightarrow y_{2}$ & - & 3 \\
Total & & 20 & 29 \\
\end{tabular}




\section{Assignment of Vehicles}

In this section, we investigate the BEPP to propose an integrated evacuation planning approach for such a problem.

\subsection{Bus-based Evacuation Planning Problem}

Problem 2. Let $i \in Y, j \in Z$ with $\tau_{i j}$ as the source-sink travel times. Let $\tau_{d i}, l_{i}$ and $\mu_{j}$ be the depot-source travel times, number of evacuees and sink capacities, respectively. Then the BEPP is to find a tour plan to minimize the maximum travel times overall buses such that all the evacuees be transported to the sink.

Let the number of evacuees at every source be known. Assume $Q$ be the uniform bus capacity, as a unit. The movement between the pickup locations $Y$ is ignored and the same situation in between the sinks $Z$. The set of tours of the buses cannot be changed anymore after they start to move. Let $\sum_{i \in Y} l_{i}$ and $\sum_{j \in Z} \mu_{j}$ be the total number of evacuees and the total sink capacity, respectively. The maximum number of rounds $R$ for the evacuation process is given by $\sum_{i \in Y} l_{i}$. The nonnegative travel cost of $\tau_{i j}$ on each edge $e=(i, j) \in E$ are taken symmetric and satisfies the triangle inequality. The variables $\tau_{t o}^{b r}$ and $\tau_{b a c k}^{b r}$ give the travel time for the bus $b$ within the round $r$ from source to sink, and from the sink to the next source, respectively. Let $\mathrm{T}_{\max }$ be the duration of evacuation overall buses. The problem can be formulated to minimize $T_{\max }$ such that,

$$
\begin{gathered}
T_{\text {max }} \geq \sum_{i \in Y} \sum_{i \in Z} \tau_{d i} x_{i j}^{b 1}+\sum_{r \in R} \tau_{t o}^{b r}+\sum_{r \in R} \tau_{\text {back }}^{b r}, \forall b \in B, \\
\tau_{t o}^{b r}=\sum_{i \in Y} \sum_{i \in Z} \tau_{i j} x_{i j}^{b r}, \forall b \in B, r \in R, \\
\tau_{b a c k}^{b r} \geq \sum \tau_{i j}\left[\sum_{k \in Y} x_{k j}^{b r}+\sum_{l \in Z} x_{i l}^{b, r+1}-1\right], \forall b \in B, r \in R-1, \\
\sum_{i \in Y} \sum_{i \in Z} x_{i j}^{b r} \geq \sum_{i \in Y} \sum_{i \in Z} x_{i j}^{b, r+1}, \forall b \in B, r \in R, \\
\sum_{i \in Y} \sum_{i \in Z} x_{i j}^{b r} \leq 1, \forall b \in B, r \in R-1, \\
\sum_{i \in Y} \sum_{i \in Z} \sum_{r \in R} x_{i j}^{b r} \geq l_{i}, \forall i \in Y, \\
\sum_{i \in Y} \sum_{b \in B} \sum_{r \in R} x_{i j}^{b r} \leq \mu_{j}, \forall j \in Z, \\
x_{i j}^{b r} \in\{0,1\}, \forall \tau_{t o}^{b r}, \tau_{b a c k}^{b r}, \mathrm{~T}_{\max } \in \mathbb{R} .
\end{gathered}
$$

Constraint (5) needs $\mathrm{T}_{\max }$ to be greater than or equal to the maximal travel cost subject to all bus movements and is to be minimized on $T_{\max }$. Constraints (6) and (7) are the measure of travel time for the bus $b$ within the round $r$ from source to sink, and from that sink to the next source, respectively. Constraint (8) tells that the tours are connected and can stop whenever they like. Constraint (9) allows a bus from a source to a sink per round. Also (10) and (11) represent the bus and shelter capacity constraints, respectively. Constraint (12) represents whether the bus $b$ travels from source $i$ to $\operatorname{sink} j$ in the round $r$.

For $\mathrm{i} \in \mathrm{Y}, \mathrm{j} \in \mathrm{Z}$. Let $\tau_{d i}, \tau_{i j}, l_{i}$ and $\mu_{j}$ as in Problem 2. For $k \in \mathbb{R}$, is there a tour plan with $\mathrm{T}_{\max }<k$, for the complete evacuation? Regarding the complexity for such a decision version, the following result is established. [22]

Theorem 5. The decision version of BEPP is NP-complete, even if $\tau_{d i}=0$ and $\tau_{i j}=\tau_{i^{\prime} j} \forall i, i^{\prime} \in Y$ and $j \in Z$.

For a solution, the branch and bound algorithms with four upper bounds and three lower bounds for time, three branching rules to minimize the number of branches and two tree reduction strategies to avoid the equivalent branches have been presented in [8]. Upper bounds have been constructed in polynomial-time complexity by four heuristic algorithms. Among the lower bounds, the first one is based on the estimation of the travel times from sources to sinks and from sinks to sources, respectively. The second lower bound is based on the fact that lower bound for the maximum travel time is the average travel time. The third one is about the simplification of model formulation.

\subsection{An Integrated Evacuation Planning Approach}

Transit-buses having uniform capacity $Q$ are assigned from $d$ which are sufficiently nearer to $Y$ in $N_{2}$ on the firstcome-first-serve basis. Such assignment begins only after $\alpha_{1} \geq Q$ for $\alpha_{1}$ be the number of evacuees arrived at the highest pickup demand. For the subsequent assignments, the effective waiting instance $\xi$ is almost negligible. However, waiting at pickup locations is comparatively better than to wait at the disaster zone itself. Buses are assumed to pick up their full capacities. For this, the potential demands of the pickup locations are adjusted to be the integral multiple of busloads. Let the potential demand of the pickup location $y_{k} \in Y$ be $\alpha\left(y_{k}\right)$. Then the demands can be adjusted to be $\alpha^{\prime}\left(y_{k}\right)$ by using the following demand adjustment,

$$
\alpha^{\prime}\left(y_{k-1}\right)=\left\lfloor\left\{\alpha\left(y_{k-1}\right)+\alpha\left(y_{k-2}\right)-\alpha^{\prime}\left(y_{k-2}\right)+\cdots+\alpha\left(y_{1}\right)-\alpha^{\prime}\left(y_{1}\right)\right\} / Q\right\rfloor \cdot Q
$$

But if the $k^{\text {th }}$ pickup location is the last one with the least priority, then it is taken as,

$$
\alpha^{\prime}\left(y_{k}\right)=\alpha\left(y_{k}\right)+\alpha\left(y_{k-1}\right)-\alpha^{\prime}\left(y_{k-1}\right)+\cdots+\alpha\left(y_{1}\right)-\alpha^{\prime}\left(y_{1}\right)
$$


With the constraints (5-12), the integrated evacuation planning problem can be reformulated to minimize $T_{\max }$ such that:

$$
T_{\text {max }} \geq \xi+\sum_{r \in R} \tau_{t o}^{b r}+\sum_{r \in R} \tau_{\text {back }}^{b r} \forall b \in B,
$$

As such an integrated problem is not easier than the BEPP in Section 4.1, we state the following result.

Theorem 6. The decision version of the integrated evacuation planning problem is NP-complete.

Example 2. By using Equation (13), the arrivals of

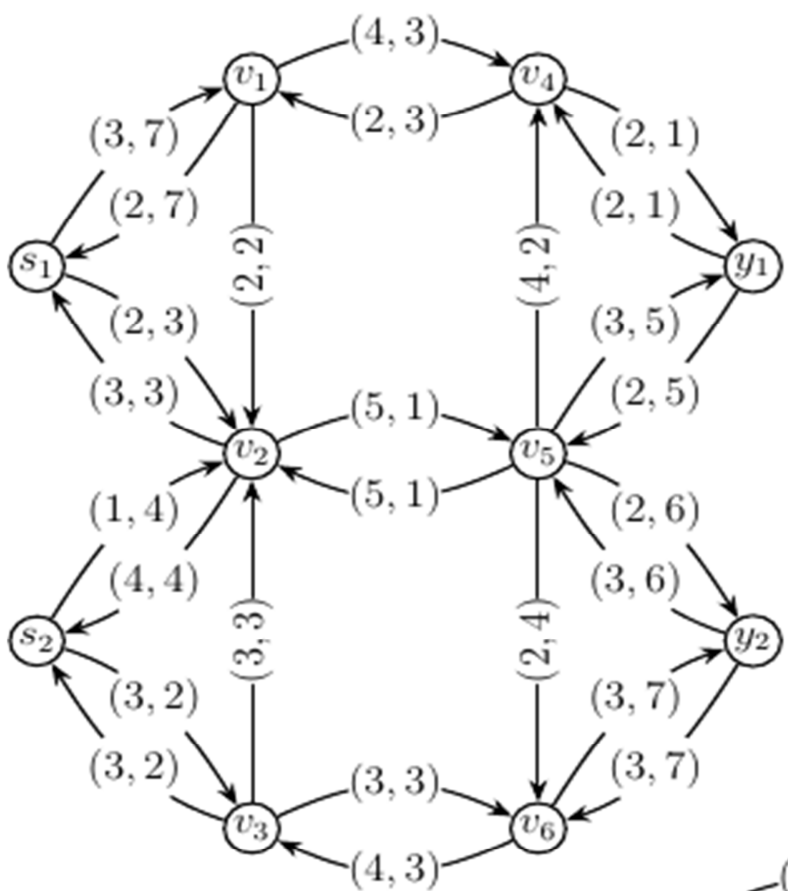

(i)

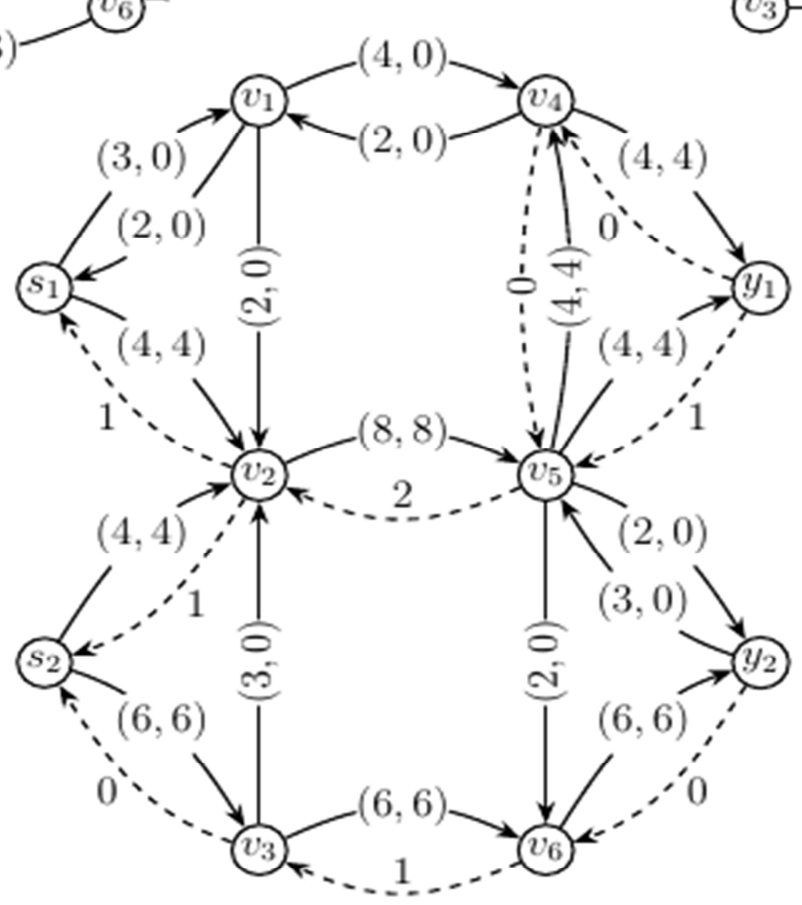

(iii) evacuees at $Y$ of $N_{1}$ in Figure 2(i) is shown as in Table 1 and Table 2 can be adjusted to be the integral multiple of busloads as in Table 3 and Table 4, with their respective paths assignment without and with arc reversal capability in $N_{1}$, respectively. Such evacuees are to be assigned on the integrated evacuation network (cf. Example 3) where the transit-buses are assumed to pick up their full capacities except for the last trip. For the last trip, it is assumed to be the integral multiple of busloads for the remaining evacuees (if any) for the complete evacuation by convention.

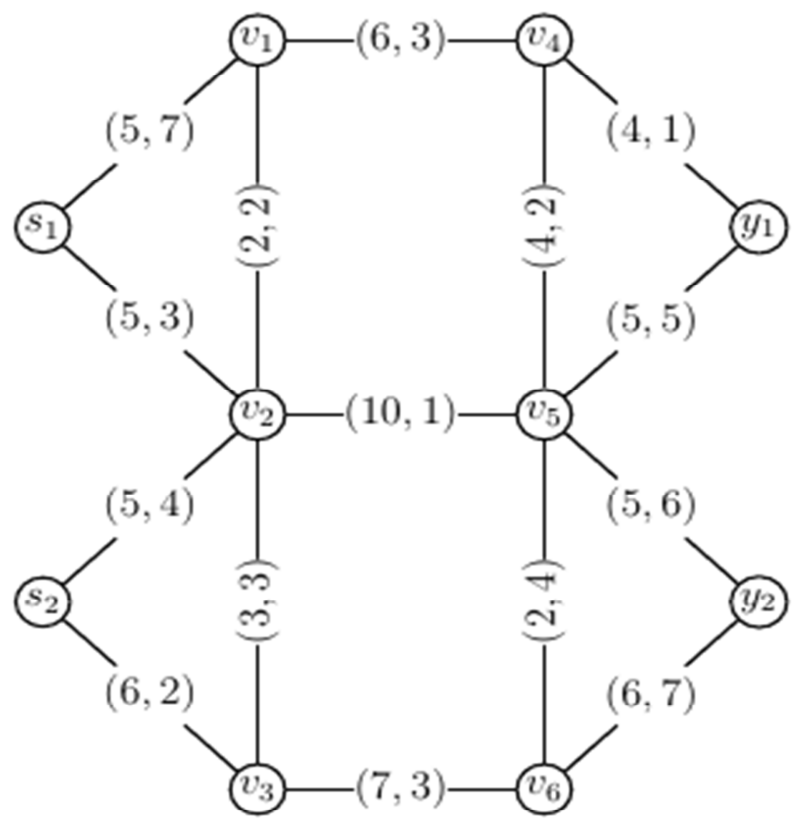

(ii)

Figure 2. (i) Primary sub-network with $\left(u_{a}, \tau_{a}\right)$, (ii) auxiliary network to (i), and (iii) network showing the arc capacities, flows and the saved arc capacities due to partial arc reversal concerning to adjusted demands. 
Table 3. The arrival of evacuees at Y after demand adjustment without partial arc reversal capability.

\begin{tabular}{|c|c|c|c|c|c|}
\hline Released time & Reached time & Paths assignment & Flow at $y_{1}$ & Flow at $y_{2}$ & Total flow \\
\hline 0 & 7 & $s_{1} \rightarrow v_{2} \rightarrow v_{5} \rightarrow v_{4} \rightarrow y_{1}$ & 2 & & 2 \\
\hline 1 & 8 & $s_{1} \rightarrow v_{2} \rightarrow v_{5} \rightarrow v_{4} \rightarrow y_{1}$ & 2 & & 4 \\
\hline 2 & 9 & $s_{1} \rightarrow v_{2} \rightarrow v_{5} \rightarrow v_{4} \rightarrow y_{1}$ & 2 & & 6 \\
\hline 0 & 10 & $s_{2} \rightarrow v_{2} \rightarrow v_{5} \rightarrow y_{1}$ & 1 & & 9 \\
\hline 4 & 11 & $s_{1} \rightarrow v_{2} \rightarrow v_{5} \rightarrow v_{4} \rightarrow y_{1}$ & 2 & & 11 \\
\hline 1 & 11 & $s_{2} \rightarrow v_{2} \rightarrow v_{5} \rightarrow y_{1}$ & 1 & & 12 \\
\hline 5 & 12 & $s_{1} \rightarrow v_{2} \rightarrow v_{5} \rightarrow v_{4} \rightarrow y_{1}$ & 2 & & 14 \\
\hline 2 & 12 & $s_{2} \rightarrow v_{2} \rightarrow v_{5} \rightarrow y_{1}$ & 1 & & 15 \\
\hline 0 & 12 & $s_{2} \rightarrow v_{3} \rightarrow v_{6} \rightarrow y_{2}$ & - & 3 & 18 \\
\hline 6 & 13 & $s_{1} \rightarrow v_{2} \rightarrow v_{5} \rightarrow v_{4} \rightarrow y_{1}$ & 2 & & 20 \\
\hline 2 & 13 & $s_{2} \rightarrow v_{2} \rightarrow v_{5} \rightarrow y_{1}$ & 1 & & 21 \\
\hline 1 & 13 & $s_{2} \rightarrow v_{3} \rightarrow v_{6} \rightarrow y_{2}$ & - & 3 & 24 \\
\hline 2 & 14 & $s_{2} \rightarrow v_{3} \rightarrow v_{6} \rightarrow y_{2}$ & - & 3 & 27 \\
\hline 3 & 15 & $s_{2} \rightarrow v_{3} \rightarrow v_{6} \rightarrow y_{2}$ & - & 2 & 29 \\
\hline Total & & & 18 & 11 & 29 \\
\hline
\end{tabular}

Table 4. The arrival of evacuees at Y after demand adjustment with partial arc reversal capability.

\begin{tabular}{lllll}
\hline Released time & Reached time & Paths assignment & Flow at $\boldsymbol{y}_{\mathbf{1}}$ & Flow at $\boldsymbol{y}_{\mathbf{2}}$ \\
\hline 0 & 7 & $s_{1} \rightarrow v_{2} \rightarrow v_{5} \rightarrow v_{4} \rightarrow y_{1}$ & 4 & \\
1 & 8 & $s_{1} \rightarrow v_{2} \rightarrow v_{5} \rightarrow v_{4} \rightarrow y_{1}$ & 4 & \\
2 & 9 & $s_{1} \rightarrow v_{2} \rightarrow v_{5} \rightarrow v_{4} \rightarrow y_{1}$ & 4 & \\
3 & 10 & $s_{1} \rightarrow v_{2} \rightarrow v_{5} \rightarrow v_{4} \rightarrow y_{1}$ & 4 & 12 \\
0 & 10 & $s_{2} \rightarrow v_{2} \rightarrow v_{5} \rightarrow y_{1}$ & 2 & 16 \\
0 & 12 & $s_{2} \rightarrow v_{3} \rightarrow v_{6} \rightarrow y_{2}$ & - & 6 \\
1 & 13 & $s_{2} \rightarrow v_{3} \rightarrow v_{6} \rightarrow y_{2}$ & - & 5 \\
Total & & & 18 & 24 \\
\hline
\end{tabular}

The partial arc reversal strategy allows the reversal of only the necessary part of the road segments along with the direction of traffic in the evacuation network, saving the remaining part of the road segments. Due to such phenomena, in some arcs, certain parts are partially reversed as necessary and some remain saving. The dotted arcs represent the arc saving along with their measure as in Figure 2(iii). Here, the road segments $\left(\mathrm{y}_{1}, \mathrm{v}_{5}\right),\left(\mathrm{v}_{5}, \mathrm{v}_{2}\right),\left(v_{2}, s_{1}\right),\left(v_{2}, s_{2}\right)$, and $\left(v_{6}, v_{3}\right)$ are saving the arc capacities of $1,2,1,1$, and 1 respectively. But no such arc saving on $\left(\mathrm{v}_{1}, \mathrm{v}_{5}\right),\left(\mathrm{y}_{1}, \mathrm{v}_{4}\right),\left(y_{2}, v_{6}\right)$ and $\left(v_{3}, s_{2}\right)$ due to their full reversal, as demanded. Saved arc capacities are beneficial for the humanitarian logistics, facility locations, and also for the emergency vehicle movements within the network during such evacuation.

\section{An Integrated Solution Approach}

In an integrated solution approach for the evacuation planning problem, the quickest transshipment of the evacuees arrived at $\mathrm{Y}$ in $\mathrm{N}_{1}$ as in the form concerning the adjusted demands are assigned to the transit-buses in the embedded network.

Algorithm 3. Evacuation planning algorithm in an integrated network topology.

Input: An embedding $N=\left(S, V, A, u_{a}, \tau_{a}, Y, d, u_{e}, \tau_{e}, Z\right)$, provided with given supply and demand.

1. Consider $N_{1}=\left(S, V, A, u_{a}, \tau_{a}, Y\right)$ having their pickup locations be $\cup y_{i}=Y$.

2. Construct a priority ordering of $Y$ assigning the highest priority to the nearest from $S$.

3. Determine the arrival of evacuees at $Y$ of $N_{1}$ from $S$ using Algorithm 2.

4. Assign the transit-buses from $d$ to $Y$ in $N_{2}=$ $\left(d, Y, u_{e}, \tau_{e}, Z\right)$ for the supplies obtained in Step 3, to the nearest $\operatorname{sink} Z$, on the first-come-first-serve basis.

5. Begin the assignment with $\alpha_{1} \geq Q$ for $\alpha_{1}$ be the collection of evacuees at $y_{1} \in Y$ and $Q$ be the homogeneous capacity of each transit-buses and is continued for the adjusted demands at $Y$ provided by Equation (13).

6. Stop, if all the supplies at each $Y$ be fulfilled, respecting the capacity constraints of $Z$.

7. Otherwise, return to Step 4

Output: Transshipment of evacuees finally to $Z$ in minimum clearance time.

Theorem 7. Algorithm 3 constructed for the evacuation planning problem in an integrated network gives the feasible solution to send the evacuees to $Z$ in minimum clearance time.

Proof. In Step $1, N_{1}$ is constructed with the pickup locations be $\cup y_{i}=Y$, and is feasible. In this prioritized subnetwork by construction, Step 2 is also feasible. The arrival of evacuees determined at $Y$ provided by Algorithm 2 gives the feasibility as well as the validity of Step 3 of Algorithm 3 . Two more Steps 4 and 5 are about the transit-buses assignment in the integrated network and are governed not only by the availability of the buses at $d$ but also by the supply available at $Y$. It is continued in $N$ for the available evacuees respecting the capacity constraints of $Z$ and are feasible. Hence, the algorithm is feasible.

Now, we show that Algorithm 3 gives the feasible solution in minimum time. The arrival of the lex-max number of evacuees at $Y$ from $S$ by using Algorithm 2 gives the quickest transshipment of evacuees in the given priority by [18] and saves the unused capacity by Theorem 3 . These resulting flows at $Y$ are taken as the input in $N_{2}$ for the required transit-buses assignment. Such assignment to nearest sink 
approach respecting the priority is with almost negligible waiting delay for these evacuees at $Y$ in the embedding. It is continued till the last evacuees are reached to the sink without violating their capacities. Hence, it gives a feasible solution with minimum clearance time. $\square$

Example 3. Let the adjusted demands as in Table 3 and Table 4 be the available supplies for the bus assignment in the embedding as shown in Figure 3. Consider the available transit-buses be $|B|=2$ as $B_{1}$ and $B_{2}$ and are with uniform capacity of 6 . The assignment concerning without and with path reversal capability in $N_{1}$ are illustrated briefly in Table 5 and Table 6, respectively. Here, the column $\theta$ represents different time instances in the integrated network, $B_{1}$ and $B_{2}$ as the assignment of transit-buses with their respective positions in $N_{2}$. Here, $M_{12}$ and $M_{21}$ are used to denote the mid-way of the road segments connecting $y_{1}$ to $z_{2}$ and $y_{2}$ to $z_{1}$ respectively. The columns for $y_{i}, y_{i}^{\prime}$ and $z_{i}$ are denoting the total flow arrived at $Y$, released from $Y$, and reached to $Z$, respectively.

The quickest transshipment at $Y$ and their assignment to $Z$ be beneficial in the network having arc reversal capability than to the network without arc reversal capability, i.e., the sooner the better. It is 14, if the partial arc reversal is allowed otherwise 17, as illustrated with respect to $\theta$. Here, the buses $B_{1}$ and $B_{2}$ have the route plan of $d \rightarrow y_{1} \rightarrow z_{1} \rightarrow$ $y_{1} \rightarrow z_{1} \rightarrow y_{2} \rightarrow z_{2} \rightarrow y_{2} \rightarrow z_{2} \quad$, and $d \rightarrow y_{1} \rightarrow z_{2}$, respectively concerning without arc reversal at $N_{1}$. But, with respect to arc reversal at $N_{1}$, the route plan of the buses are $d \rightarrow y_{1} \rightarrow z_{1} \rightarrow y_{1} \rightarrow z_{2} \rightarrow y_{2} \rightarrow z_{2}$ and $d \rightarrow y_{1} \rightarrow z_{1} \rightarrow$ $y_{2} \rightarrow z_{2}$, respectively.

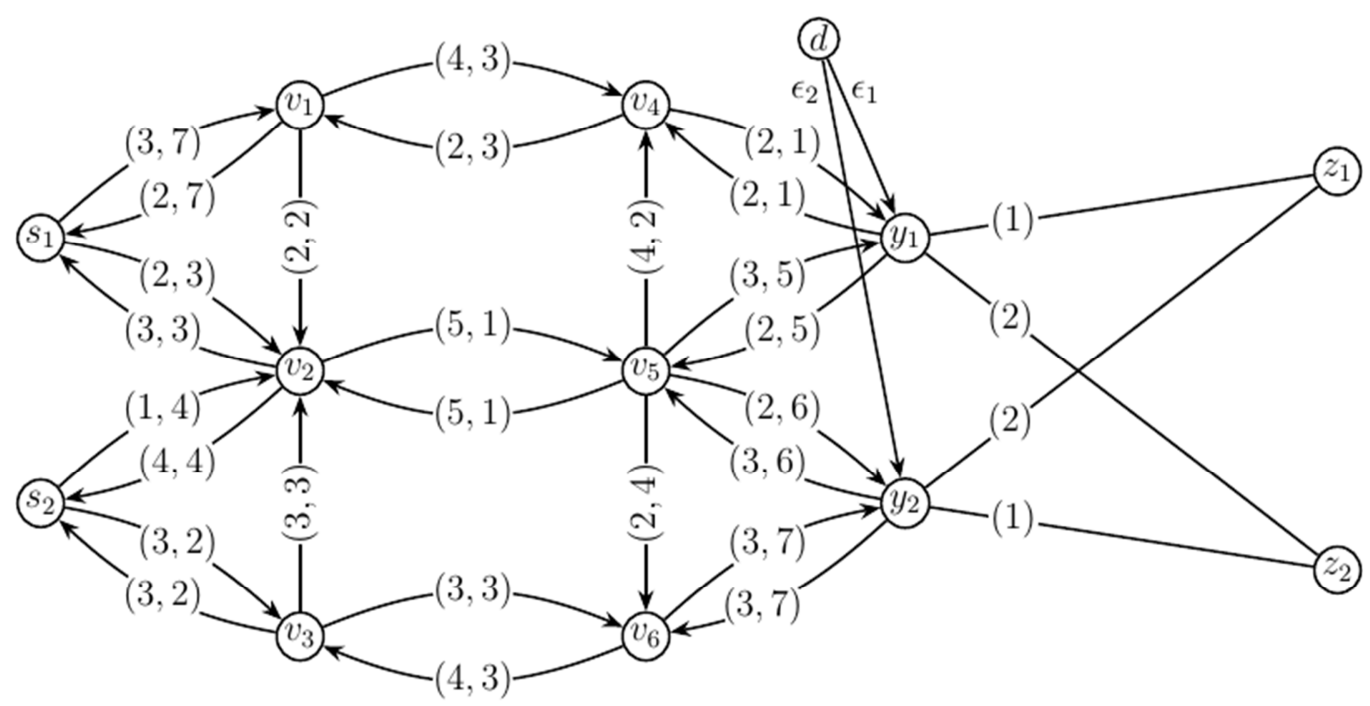

Figure 3. An embedding $N=N_{1} \cup N_{2}$ showing an instance of integrated evacuation scenario.

Table 5. Integrated evacuation planning approach in $N$ for the demand adjusted evacuees.

\begin{tabular}{lllllllll}
\hline $\boldsymbol{\theta}$ & $\boldsymbol{B}_{\mathbf{1}}$ & $\boldsymbol{B}_{\mathbf{2}}$ & $\boldsymbol{y}_{\mathbf{1}}$ & $\boldsymbol{y}_{\mathbf{1}}^{\prime}$ & $\boldsymbol{y}_{\mathbf{2}}$ & $\boldsymbol{y}_{\mathbf{2}}^{\prime}$ & $\boldsymbol{z}_{\mathbf{1}}$ & $\boldsymbol{z}_{\mathbf{2}}$ \\
\hline 9 & $y_{1}$ & & 6 & 6 & & & & \\
10 & $z_{1}$ & & 9 & & & & 6 & \\
11 & $y_{1}$ & & 12 & 12 & & & & \\
12 & $z_{1}$ & & 15 & & 3 & & 12 & \\
13 & $M_{21}$ & $y_{1}$ & 18 & 18 & 6 & & & \\
14 & $y_{2}$ & $\mathrm{M}_{12}$ & & & 9 & 6 & & \\
15 & $z_{2}$ & $z_{2}$ & & & 11 & & & 12 \\
16 & $y_{2}$ & & & & & 11 & & \\
17 & $z_{2}$ & & & & & & & 17 \\
\hline
\end{tabular}

Table 6. Integrated evacuation planning approach in $N$ for the demand adjusted evacuees with partial arc reversal.

\begin{tabular}{lllllllll}
\hline $\boldsymbol{\theta}$ & $\boldsymbol{B}_{\mathbf{1}}$ & $\boldsymbol{B}_{\mathbf{2}}$ & $\boldsymbol{y}_{\mathbf{1}}$ & $\boldsymbol{y}_{\mathbf{1}}^{\prime}$ & $\boldsymbol{y}_{\mathbf{2}}$ & $\boldsymbol{y}_{\mathbf{2}}^{\prime}$ & $\boldsymbol{z}_{\mathbf{1}}$ & $\boldsymbol{z}_{\mathbf{2}}$ \\
\hline 8 & $y_{1}$ & & 8 & 6 & & & & \\
9 & $z_{1}$ & $y_{1}$ & 12 & 12 & & & 6 & \\
10 & $y_{1}$ & $z_{1}$ & 18 & 18 & & & 12 & \\
11 & $\mathrm{M}_{12}$ & $M_{21}$ & & & 6 & 6 & & \\
12 & $z_{2}$ & $y_{2}$ & & & 11 & 11 & & 6 \\
13 & $y_{2}$ & $z_{2}$ & & & & & & 12 \\
14 & $z_{2}$ & & & & & & & 17 \\
\hline
\end{tabular}

\section{Conclusions}

The quickest transshipment problem is to find the minimum clearance time to send a given amount of flow from multiple sources to multiple sinks network. For a lexmax dynamic flow problem, we are given a time horizon and a dynamic network with an ordered set of terminals, where we need a feasible dynamic flow that lexicographically maximizes the flow amount leaving each terminal in the given priority. The quickest transshipment problem in a dynamic network can be reduced to an equivalent lex-max flow problem and is solved in polynomial-time complexity.

Evacuees are collected at the prioritized pickup locations of the primary sub-network following the quickest transshipment in the lex-max flow approach and are assigned simultaneously to the homogeneous transit-buses in the secondary sub-network on the first-come-first-serve basis. The waiting delay at the pickup locations is almost negligible. On the other side, if there is some waiting, it is preferable to wait at such pickup locations rather than to be at the danger regions. The arc reversal capability of the primary 
sub-network is beneficial to improve the minimum clearance time of the evacuees in the integrated evacuation network where the saved unused arc capacities are useful for emergency facility locations and logistics. It is interesting to extend these techniques for the heterogeneous or mixed model transit-buses and also for the disparate group of evacuees in a different network topology.

\section{Acknowledgements}

The first author wants to acknowledge the University Grants Commission, Nepal, for the $\mathrm{PhD}$ research fellowship award. The authors would also like to thank the anonymous referees and the editor for their valuable suggestions to improve the quality of this paper.

\section{References}

[1] Ford, L. R. and Fulkerson, D. R. (1958). Constructing maximal dynamic flows from static flows. Operations Research, 6: 419-433.

[2] Ford, L. R. and Fulkerson, D. R. Flows in Networks. Princeton University Press, Princeton, NJ, 1962.

[3] Adhikari, I. M., Pyakurel U., and Dhamala, T. N. (2020). An integrated solution approach for the time minimization evacuation planning problem. International Journal of Operation Research, 17 (1): 27-39.

[4] Dhamala, T. N. and Adhikari, I. M. (2018). On evacuation planning optimization problems from transit-based perspective. International Journal of Operation Research, 15 (1): 29-47.

[5] Dhamala, T. N., Adhikari, I. M., Nath, H. N., and Pyakurel, U. (2018). Meaningfulness of OR models and solution strategies for emergency planning. In: Living Under the Threat of Earthquakes, Edited by Kruhl, J. H., Adhikari, R. and Dorka, U. E., Springer Natural Hazards, 175-194.

[6] Dhamala, T. N., Pyakurel, U., and Dempe, S. (2018). A critical survey on the network optimization algorithms for evacuation planning problems. International Journal of Operation Research, 15 (3): 101-133.

[7] Bish, D. R. (2011). Planning for a bus-based evacuation. OR Spectrum, 33: 629-654.

[8] Goerigk, M., Grün B., and He $\mathrm{e}_{\beta}$ ler, P. (2013). Branch and bound algorithms for the bus evacuation problem. Computers and Operations Research, 40: 3010-3020.
[9] Pyakurel, U., Goerigk, M., Dhamala, T. N., and Hamacher, H. W. (2015). Transit dependent evacuation planning for Kathmandu valley: a case study. International Journal of Operations Research Nepal, 5: 49-73.

[10] Megiddo, N. (1974). Optimal flows in networks with multiple sources and sinks. Mathematical Programming, 7: 97-107.

[11] Megiddo, N. (1977). A good algorithm for lexicographically optimal flows in multi-terminal networks. Bulletin of the American Mathematical Society, 83: 407-409.

[12] Minieka, E. Maximal. (1973). Lexicographic, and dynamic network flows. Operation Research, 21: 517-527.

[13] Wilkinson, W. L. (1971). An algorithm for universal maximal dynamic flows in a network. Operations Research, 19 (7): 1602-1612.

[14] Kamiyama, N. (2019). Lexicographically optimal earliest arrival flows. Networks, 1-16.

[15] Pyakurel, U. and Dhamala, T. N. (2015). Models and algorithms on contraflow evacuation planning network problems. International Journal of Operations Research, 12 (2): 36-46.

[16] Pyakurel, U., Nath, H. N., and Dhamala, T. N. (2019). Partial contraflow with path reversals for evacuation planning. Annals of Operation Research, 283, 591-612.

[17] Pyakurel, U., Nath, H. N., Dempe, S., and Dhamala, T. N. (2019). Efficient dynamic flow algorithms for evacuation planning problems with partial lane reversal. Mathematics, 7 (10), 993.

[18] Hoppe B. and Tardos E. (2000). The quickest transshipment problem. Mathematics of Operation Research, 25 (1): 36-62.

[19] Fleischer L. K. (2001). Faster algorithms for the quickest transshipment problem. SIAM Journal on Optimization, 12 (1): 18-35.

[20] Orlin, J. B. (1988). Faster strongly polynomial cost flow algorithm. Proceeding of the 20th annual symposium of theory of computing, 377-387.

[21] Schloter, M., and Skutella, M. (2017). Fast and MemoryEfficient Algorithms for Evacuation Problems. Proceedings of the Twenty-Eighth Annual ACM-SIAM Symposium on Discrete Algorithms, 821-840.

[22] Goerigk, M. and Grün B. (2014) A robust bus evacuation model with delayed scenario information. OR Spectrum, 36: 923-948. 